\section{MS39-P7 Precise determination of lattice constants by energy dispersive Laue experiments with a PILATUS detector} Kurdzesau Fiodar ${ }^{1}$, Schneebeli Matthias $^{2}$, Pilipp Volker ${ }^{2}$, Mueller
Marcus ${ }^{2}$, Weber Thomas

1. Laboratorium für Kristallographie, ETH Zurich, Vladimir-Prelog-Weg 5, Zurich 8093, Switzerland

2. DECTRIS Ltd., Neuenhofstrasse 107, Baden 5400, Switzerland

email: fiodar.kurdzesau@mat.ethz.ch

The most common application of traditional (energy integrative) Laue technique is certainly identification of crystal orientations [1]. For obtaining further information such as lattice constants or integral Bragg intensities complementary experiments are required, which frequently cannot be performed without violating the integrity of bulky samples or moving the crystals. The later is of particular nuisance in cases of non-ambient experiments where the possibility of stationary measurements would heavily simplify experimental setups. Modern energy discriminating pixel detectors (e.g. PILATUS [2], frame store pnCCD [3] etc.) allow overcoming this problem providing the full information about bulky and/or stationary crystals via energy-dispersive Laue diffraction (EDLD) experiments.

In this study EDLD measurements are performed with a four-circle Eulerian geometry diffractometer equipped with a conventional X-ray source (Mo sealed tube) and a PILATUS $300 \mathrm{~K}$ detector [2]. The flexibility of the diffractometer is utilized for testing various experimental configurations (forward, back scattering etc.), while the crystal itself was not moved during the experiments. Parallax measurements of primary and diffracted radiation are used for determining experimental errors directly with the crystal under investigation, which allow correcting unavoidable misalignments of large samples. The PILATUS detector's energy resolution $(<0.12 \mathrm{keV})$ is sufficient for a proper determination of the scattering vector lengths of recorded reflections, i.e. three-dimensional reciprocal space reconstructions, where the orientation and lattice constant determination become possible following standard procedures [4].

The developed technique was tested with several bulky samples (silicon, quartz, $\mathrm{MgZn}_{2}$ etc.). The crystal orientation and lattice parameters could be identified with a satisfying precision $\left(0.05^{0}\right.$ and $0.01 \AA$, or better). Possible applications of this method and its further developments (quantitative structure determination of stationary and/or bulky crystals) will be discussed.

[1] Z. Ren, D. Bourgeois, J.R. Helliwell et al, J. Synchrotron Rad., 1999, 6, 891-917

[2] P. Kraft, A. Bergamaschi, Ch. Brönnimann et al, IEEE Trans.Nucl.Sci., 2009, 56(3), 758-764

[3] S. Send, M. von Kozierowski, T. Panzner et al, J.Appl.Cryst., 2009, 42, 1139-1146

[4] W. Kabsch, J.Appl.Cryst., 1988, 21, 916-924

Keywords: Energy-dispersive X-ray diffraction analysis, Laue diffraction, PILATUS detectors.
MS39-P8 ISX stage: from automated in situ screening to structure solution

Severine Freisz ${ }^{1}$, Freisz Severine ${ }^{1}$, Benning Matthew ${ }^{2}$

1. Bruker AXS GmbH Oestliche Rheinbrueckenstrasse 49, 76187 Karlsruhe, Germany

2. Bruker AXS Inc. 5465 E. Cheryl Parkway Madison, WI 53711 United States

email: severine.freisz@bruker.com

Recent developments in hardware and software are greatly increasing the capabilities of in-house diffraction systems, making it more routine to obtain de novo structural information in the home lab. We have now introduced the D8-VENTURE solution for structural biology with the PHOTON 100 detector, featuring the first CMOS active pixel sensor for X-ray crystallography. Our new microfocus source, the METALJET delivers beam intensity exceeding those of typical bending-magnet beamlines.

Very recently, we developed a fully automated solution for in situ crystal screening in plates, the ISX stage. This stage facilitates the investigation of a large number of crystals within the routine workflow of the crystallization lab.

The combination of the sensitive PHOTON 100 detector and a high-intensity X-ray source maximizes the diffraction signal from even the smallest, most weakly diffracting crystals. The ISX software provides an intuitive user environment to maximize the productivity of even occasional users.

The system can be quickly converted from the typical single-crystal diffractometer to the completely motorized in situ crystal screening device, leaving the KAPPA untouched. The system can be reverted to the standard diffractometer just as quickly, after a favorite crystal has been identified for data collection.

Here we present all the ISX stage features and data we collected in house.

Keywords: In situ screening, Structural Biology 